\title{
Cytokine and chemokine signatures associated with hepatitis B surface antigen loss in hepatitis B patients
}

\author{
Sachiyo Yoshio, ${ }^{1}$ Yohei Mano, ${ }^{1}$ Hiroyoshi Doi, ${ }^{1}$ Hirotaka Shoji, ${ }^{1}$ Tomonari Shimagaki, ${ }^{1}$ \\ Yuzuru Sakamoto, ${ }^{1}$ Hironari Kawai, ${ }^{1}$ Michitaka Matsuda, ${ }^{1}$ Taizo Mori, ${ }^{1}$ Yosuke Osawa, ${ }^{1}$ \\ Masaaki Korenaga, ${ }^{1}$ Masaya Sugiyama, ${ }^{2}$ Masashi Mizokami, ${ }^{2}$ Eiji Mita, ${ }^{3}$ Keiko Katayama, ${ }^{4}$ \\ Junko Tanaka, ${ }^{4}$ and Tatsuya Kanto ${ }^{1}$ \\ 'Department of Liver Disease, Research Center for Hepatitis and Immunology, National Center for Global Health and \\ Medicine, Kohnodai, Ichikawa, Japan. 'Department of Genome Medical Sciences Project, Research Institute, National \\ Center for Global Health and Medicine, Ichikawa, Japan. ${ }^{3}$ Department of Gastroenterology and Hepatology, National \\ Hospital Organization, Osaka National Hospital, Hoenzaka, Chuo-ku, Osaka, Japan. ${ }^{4}$ Department of Epidemiology, \\ Infectious Disease Control and Prevention, Institute of Biomedical and Health Sciences, Hiroshima University, \\ Minami-ku, Hiroshima, Japan.
}

BACKGROUND. The clearance of hepatitis B surface antigen ( $\mathrm{HBsAg}$ ) loss, defined as functional cure, is a clinical target in patients with chronic hepatitis B (CH). To understand the immune responses underlying functional cure, we evaluated cytokine and chemokine expression profiles from patients with resolving and nonresolving acute hepatitis $B(A H)$.

METHODS. We cross-sectionally evaluated 41 chemokines and cytokines at the peak of hepatitis in the sera from 41 self-limited AH patients who achieved $\mathrm{HBsAg}$ seroconversion, $8 \mathrm{AH}$ patients who failed to clear HBsAg within 1 year after the diagnosis, $8 \mathrm{CH}$ patients with hepatic flare, and 14 healthy volunteers. We longitudinally examined 41 chemokines and cytokines in the sera from 4 self-limited AH patients, 3 chimpanzees inoculated with hepatitis B virus (HBV), and 2 CH patients treated with nucleotide analogs and PEG-IFN- $\alpha$, one resulting in functional cure.

RESULTS. In AH patients and HBV-inoculated chimpanzees with HBsAg loss, CXCL9, CXCL10, CXCL11, CXCL13, and IL-21 were elevated at hepatitis with subsequent decline of HBsAg. Interestingly, IL-21 elevation was observed only in resolving $\mathrm{AH}$ patients but not in nonresolvers. CXCL13 and IL-21 elevation was not observed in CH patients who failed to attain HBsAg loss, even at hepatic flare. A concomitant increase of CXCL13 and IL-21 was significant in CH patients who attained HBsAg seroconversion with a sequential therapy.

CONCLUSION. Elevation of serum CXCL9, CXCL10, CXCL11, CXCL13, and IL-21 might be a hallmark of functional cure of $\mathrm{AH}$ or $\mathrm{CH}$ patients.

Conflict of interest: The authors have declared that no conflict of interest exists.

Submitted: May 15, 2018

Accepted: September 4, 2018 Published: October 18, 2018

Reference information: JCI Insight. 2018;3(20):e122268. https://doi.org/10.1172/jici. insight.122268.

\section{Introduction}

It is estimated that 257 million people globally, or $3.5 \%$ of the population, were living with chronic hepatitis B virus (HBV) infections in 2015 (1). Left untreated, HBV can lead to cirrhosis and hepatocellular carcinoma in approximately $20 \%$ of infected patients. To prevent or reduce the risk of life-threatening complications, eradication of HBV from chronically infected people is desired. Hepatitis B surface antigen (HBsAg) is regarded as a surrogate marker of HBV covalently closed circular DNA (cccDNA) in infected hepatocytes. HBsAg clearance is a clinical target for risk reduction of hepatocellular carcinoma. However, in patients with chronic $\mathrm{HBV}(\mathrm{CH})$ infection, the probability of spontaneous HBV eradication is less than $1 \%$ per year, and HBsAg loss is achieved by PEG-IFN- $\alpha$ and/or nucleos(t)ide analog (NA) treatments in $9 \%$ after a 5 -year follow-up (2-4). It is thus critical to develop new antiviral strategies for achieving a realistic functional cure of $\mathrm{CH}$ infection (HBsAg loss with/without anti-HBs Ab seroconversion) $(5,6)$.

Primary HBV infection is self-limited in more than $95 \%$ of immunocompetent adults, while $\mathrm{CH}$ patients rarely attain spontaneous HBsAg clearance. Cumulative reports showed that immune response against 
$\mathrm{HBV}$ is downregulated in patients with $\mathrm{CH}$, probably due to the exhausted phenotype of $\mathrm{T}$ cells. Regardless of such difference, extensive studies on the immune control of $\mathrm{HBV}$ at primary infection would provide insights into the fundamental mechanisms of HBsAg loss. A significant impact of IFN- $\alpha$ or TNF- $\alpha$ on the reduction of HBV or cccDNA levels has been reported (7), providing a proof of concept for immune-modulating therapy. The important roles of $\mathrm{HBV}$-specific $\mathrm{CD} 4^{+}$and $\mathrm{CD} 8^{+} \mathrm{T}$ cells in $\mathrm{HBV}$ clearance have also been well demonstrated by studies enrolling acute hepatitis B (AH) patients or HBV-infected chimpanzees (8-11). Moreover, Fisicaro et al. reported that NK cells are activated prior to the increase of Ag-specific $\mathrm{CD}^{+}$or $\mathrm{CD}^{+} \mathrm{T}$ cells in blood donors who cleared $\mathrm{HBV}$ without apparent alanine aminotransferase (ALT) elevation (12). We recently reported that CXCR3 ligands (CXCL9, CXCL10, and CXCL11) are robustly elevated at the peak of ALT elevation in patients with AH, being induced by coexisting NK cells and plasmacytoid DCs (pDCs) in response to $\operatorname{HBV}(12,13)$. Macrophages have also been drawing much attention as a key player in the prevention of HBV transmission and replication (14). These data suggest that the coordinated activation of innate and adaptive immune cells is key to successful anti-HBV responses at primary infection. However, the underlying mechanisms linking the innate and adaptive immune systems in a timely manner, in light of the clinical course of hepatitis, have largely remained undetermined.

In this study, in order to gain insight into the mechanisms of functional cure in patients with $\mathrm{CH}$, we intended to clarify the machinery of the immune response that underscores resolving $\mathrm{AH}$. To this end, we comprehensively analyzed chemokine dynamics in different cohorts: patients with $\mathrm{AH}$ or $\mathrm{CH}$, and HBVinoculated chimpanzees. Longitudinal and cross-sectional analyses of the subjects revealed that the timely activation of chemokines could lead to subsequent immune reactions, probably by mobilizing the relevant immune cells. Simultaneous increase of CXCL9, CXCL10, CXCL11, CXCL13, and IL-21 at hepatitis is of significance in resolving patients with $\mathrm{AH}$.

\section{Results}

CXCL9, CXCL10, CXCL11, CXCL13, and IL-21 at ALT peak were higher in resolving AH patients than those in CH patients with persistent infection. We examined 41 chemokines and cytokines in the sera from $49 \mathrm{AH}$ patients (41 self-limited $\mathrm{AH}[\mathrm{sAH}]$ and $8 \mathrm{AH}$ patients with persistence $[\mathrm{pAH}]), 8 \mathrm{CH}$ patients with hepatic flare, and 14 healthy volunteers (HVs) (Table 1). CX3CL1, CXCL1, CXCL9, CXCL10, CXCL11, CXCL12, CXCL13, CCL1, CCL3, CCL7, CCL8, CCL11, CCL13, CCL15, CCL17, CCL20, CCL24, CCL25, IL-1ß, IL-2, IL-4, IL-6, IL-8, IL-21, IFN- $\gamma$, and TNF- $\alpha$ were significantly higher in HBV-infected patients (sAH, $\mathrm{pAH}$, and $\mathrm{CH}$ ) than those in HVs (data not shown). To understand the immune responses underlying HBsAg loss, we compared the serum levels of chemokines and cytokines between the sAH group with HBsAg clearance and the $\mathrm{CH}$ group at flare with persistent infection. Among 41 chemokines and cytokines, CXCL9, CXCL10, CXCL11, CXCL13, and IL-21 were significantly higher in the sAH group than those in the $\mathrm{CH}$ group at flare (Figure 1A). These data suggest that dysregulation of secretion of CXCL9, CXCL10, CXCL11, CXCL13, and IL-21 might be associated with persistent infection in $\mathrm{CH}$ patients. Serum CXCL9, CXCL10, CXCL11, CXCL13, and IL-21 levels for AH patients were comparable among the patients with genotype A, B, and C (Supplemental Figure 1; supplemental material available online with this article; https://doi.org/10.1172/jci.insight.122268DS1).

$I L-21$ was elevated more vigorously in $s A H$ patients than in $p A H$ patients. To identify the factor determining spontaneous clearance in AH infection, we compared 41 chemokines and cytokines at the peak of ALT between the $\mathrm{sAH}(n=41)$ and pAH $(n=8)$ group. Interestingly, the levels of IL-21 were significantly higher in the sAH than those in the pAH (Figure 1A). CXCL9 tended to be higher in the sAH than in the pAH $(P=0.07)$ (Figure 1A), whereas CXCL10, CXCL11, and CXCL13 were comparable between them (Figure 1A). The ALT levels were higher in the sAH than those in the pAH (Figure 1A). The HBV DNA and HBsAg levels were lower in the sAH than those in the pAH (Figure 1A). These data suggest that higher levels of IL-21 and ALT, and lower HBV DNA and HBsAg levels are related to HBV resolution in patients with primary $\mathrm{HBV}$ infection.

We performed a focused analysis of chemokines and cytokines between HBeAg-positive/anti-HBenegative sAH cases $(n=18)$ and pAH cases $(n=8)$, because HBeAg-positive/anti-HBeAg-positive $(n=16)$ or HBeAg-negative/anti-HBe-positive $(n=7) \mathrm{sAH}$ patients might be in the recovering phase of AH. Serum IL-21 levels were significantly higher in the HBeAg-positive/anti-HBe-negative sAH than in pAH patients (Supplemental Figure 2A). In addition, serum CXCL9 and CXCL10 levels were significantly higher in the HBeAg-positive/anti-HBe-negative sAH than in the pAH group, while CXCL11 levels tended to be 
Table 1. Clinical background of the participants in the cross-sectional analyses

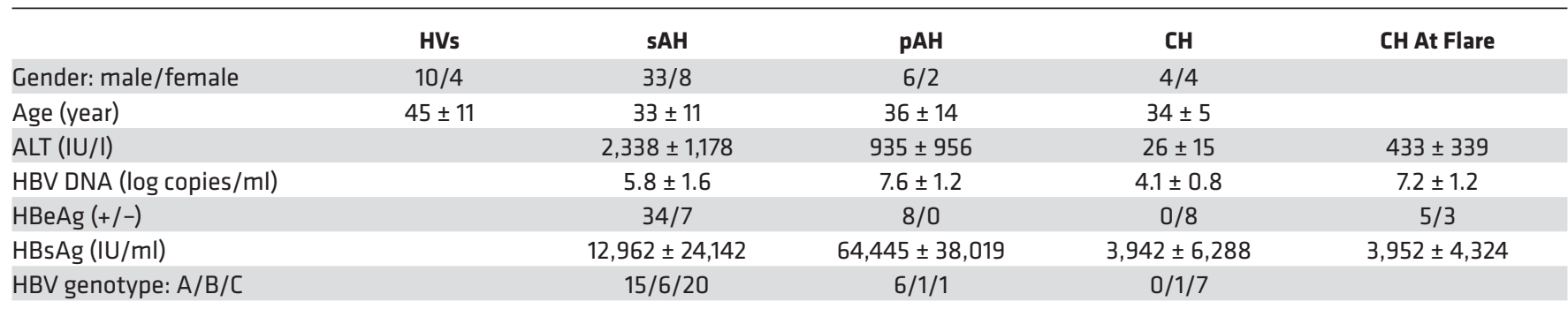

All patients were negative for anti-HCV, anti-HDV, and anti-HIV Abs, and had no apparent history of autoimmune or malignant diseases. Values are expressed as the mean $\pm \mathrm{SD}$. HVs, healthy volunteers; SAH, self-limited acute hepatitis patients; pAH, acute hepatitis patients with persistence; $\mathrm{CH}$, chronic hepatitis patients.

higher in the sAH group (Supplemental Figure 2A). These results suggest that serum CXCL9, CXLC10, and CXCL11 levels, as well as IL-21, ALT, HBV DNA, and HBsAg at the peak of ALT, could be related to $\mathrm{HBV}$ resolution in patients with primary HBV infection.

Next, we compared chemokines and clinical markers among the sAH patients with different $\mathrm{HBeAg} /$ anti-HBe status, such as HBeAg-positive/anti-HBe-negative $(n=18)$, HBeAg-positive/anti-HBe-positive $(n=16)$, and HBeAg-negative/anti-HBe-positive $(n=7)$ cases. Serum CXCL9, CXCL13, HBV DNA, and HBsAg levels were higher in the HBeAg-positive/anti-HBe-negative sAH group than those in the HBeAgnegative/anti-HBe-positive sAH group (Supplemental Figure 2B), suggesting that CXCL9 and CXCL13 might be induced at the early phase of HBV infection. In CH patients at flare, serum CXCL9, CXCL10, CXCL11, and CXCL13 levels were comparable between HBeAg-positive and -negative cases (Supplemental Figure 2C). Therefore, HBeAg status is related to the phase of acute HBV infection, thereby involving higher levels of certain chemokines/cytokines. In contrast, in the $\mathrm{CH}$ group, certain chemokines/cytokines were comparable regardless of $\mathrm{HBeAg}$ status.

Serum IL-21 levels had an inverse correlation with HBSAg levels in AH patients. We examined whether IL-21, CXCL9, CXCL10, CXCL11, and CXCL13 were correlated or not with HBV markers and ALT levels. Serum IL-21 levels at the ALT peak had an inverse correlation with HBsAg levels in AH patients (sAH + pAH) (Figure 1B), but not with HBV DNA titers (Figure 1B). Serum CXCL13 levels at the ALT peak were positively correlated with HBV DNA levels, but not with HBsAg in AH patients (sAH + pAH) (Figure 1C). Serum CXCL9, CXCL10, and CXCL11 levels had no correlations with HBsAg and HBV DNA titers (data not shown). The levels of CXCL9, CXCL11, and IL-21 at the ALT peak had a weak positive correlation with ALT levels (Figure 1D). These results suggest that CXCL9, CXCL11, and IL-21 are partly induced by liver inflammation and CXCL13 is partly induced by HBV DNA, respectively. Inverse correlation between IL-21 and HBsAg levels in AH cases raises the possibility of IL-21 playing a substantial role in HBsAg reduction.

CXCL9, CXCL10, CXCL11, CXCL13, and IL-21 were increased at the peak of ALT elevation in sAH patients. We longitudinally analyzed 41 chemokines and cytokines in 4 sAH patients (Table 2). CXCL9, CXCL10, CXCL11, CXCL13, and IL-21 increased at the peak of ALT elevation and thereafter promptly decreased together with a decrease in HBV DNA (Figure 2). The other factors showed no fixed trend throughout the course (Supplemental Figure 3, A-D). These data indicate that these factors are induced in response to HBV infection or hepatitis.

CXCL9, CXCL10, CXCL11, CXCL13, and IL-21 were elevated in chimpanzees with primary HBV infection. To understand the kinetics of immune responses from the HBV incubation phase to clearance of HBsAg, we examined 41 chemokines and cytokines in $3 \mathrm{HBV}$-inoculated chimpanzees (Ch1-3) (Table 3). Ch1, Ch2, and $\mathrm{Ch} 3$ were sacrificed at week 34, 38, and 54, respectively. The early resolvers (Ch1 and Ch2) attained HBsAg loss at week 19 and 20, respectively, while the late resolver (Ch3) did at week 50. Ch1 and Ch2 obtained anti-HBsAb at week 20 and 27, respectively, while Ch3 failed to do so throughout the observation period until week 54. In the early resolvers ( $\mathrm{Ch} 1$ and $\mathrm{Ch} 2)$, at the peak of ALT or HBV DNA elevation, CXCL9, CXCL10, CXCL11, CXCL13, and IL-21 were elevated and thereafter decreased together with HBV DNA (Figure 3). The elevation of CXCL9, CXCL10, and CXCL11 was small and no elevation of CXCL13 and IL-21 was observed in the late resolver (Ch3) (Figure 3). The other factors showed no fixed trend throughout 
A $\quad$ CXCL9

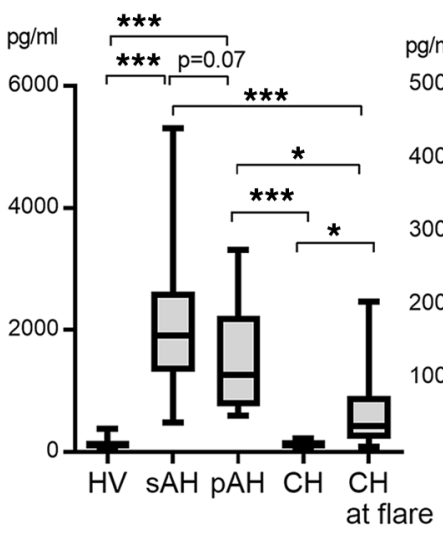

CXCL10

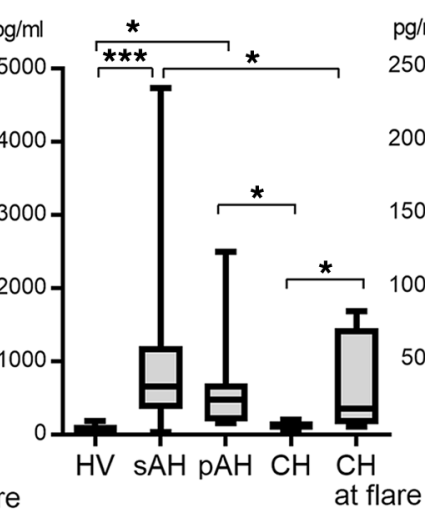

CXCL11

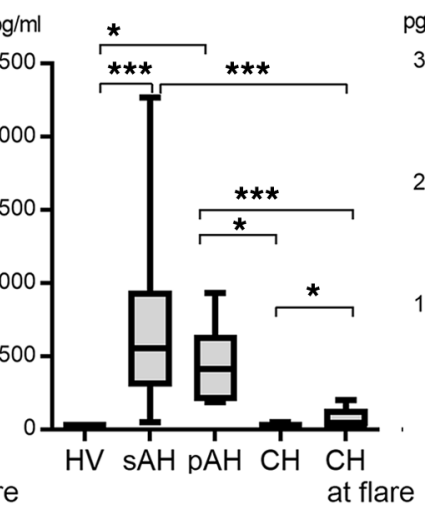

CXCL13

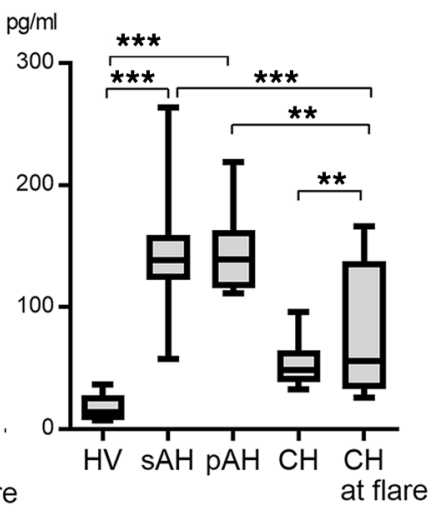

at flare
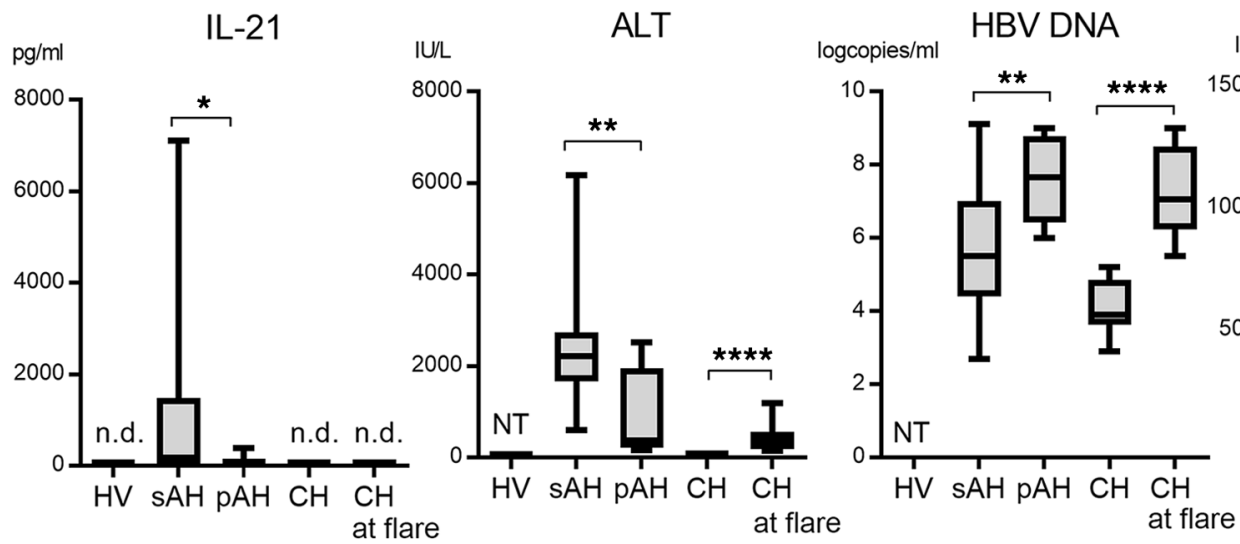

IU/mI HBsAg

B
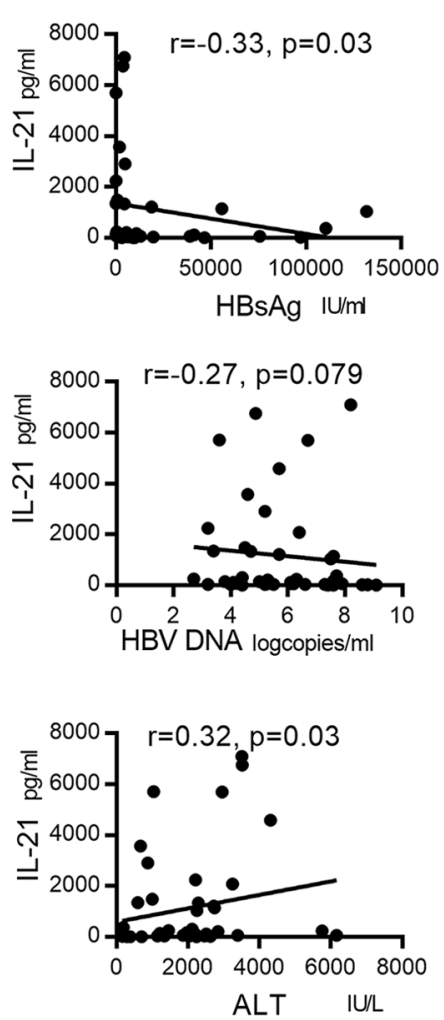

C
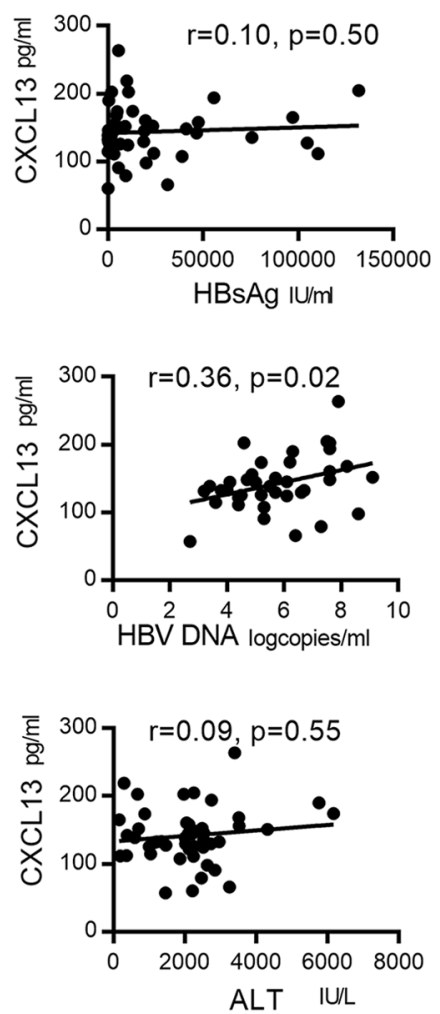

D
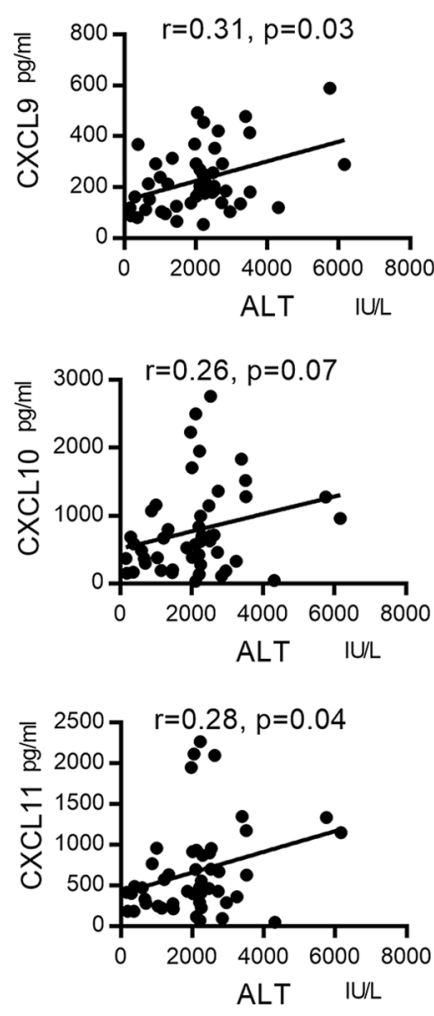
Figure 1. The comparison of serum CXCL9, CXCL10, CXCL11, CXCL13, and IL-21 levels for the patients with acute or chronic HBV infection. (A) Serum CXCL9, CXCL10, CXCL11, CXCL13, IL-21, ALT, HBV DNA, and HBsAg levels levels are shown. For patients with sAH, pAH, or CH at flare, samples obtained at the peak of alanine aminotransferase (ALT) elevation were analyzed. HV $(n=14)$, healthy volunteers; SAH $(n=41)$, self-limited acute hepatitis B patients; pAH $(n=8)$, acute hepatitis B patients who fail to clear HBsAg for more than 12 months after primary HBV infection; CH $(n=8)$, chronic hepatitis B patients; n.d., not detected; NT, not tested. Box-and-whisker plots show median, lower and upper quartiles, and minimum and maximum values. ${ }^{*} P<$ $0.05,{ }^{* *} P<0.01,{ }^{* *} P<0.001,{ }^{* * *} P<0.0001$ by Kruskal-Wallis test. (B) Correlations between IL-21 and serum HBsAg, HBV DNA, and ALT levels at the peak of ALT elevation in AH patients (including the sAH and pAH groups). (C) Correlations between CXCL13 and serum HBsAg, HBV DNA, and ALT levels at the peak of ALT elevation in AH patients (including the SAH and pAH groups). (D) Correlations between serum CXCL9, CXCL10, CXCL11, and ALT levels at the peak of ALT elevation in AH patients (including the SAH and pAH groups). The values for Spearman's correlation coefficient are given in B-D.

the course (Supplemental Figure 4, A-C). Thus, the simultaneous upregulation of CXCL9, CXCL10, CXCL11, CXCL13, and IL-21 was also associated with HBV resolution in the chimpanzees.

CXCL13 and IL-21 were elevated in CH patients who attained HBsAg loss with a sequential therapy. In order to gain insight into the roles of chemokines in HBsAg loss from patients with $\mathrm{CH}$, we examined 2 cases who had been treated with a sequential combination of NAs followed by PEG-IFN- $\alpha$. Case S1 (50-year-old male) attained HBsAg seroconversion in the follow-up after the treatment (Figure 4). During NA treatment, CXCL9 was transiently increased. Following PEG-IFN- $\alpha$ treatment, CXCL10 and CXCL11 were increased. Of particular interest, CXCL13 and IL-21 showed the peak at the point of HBsAg loss and decreased after the cessation of PEG-IFN- $\alpha$. Subsequently, case S1 gained anti-HBs antibody (Figure 4). In clear contrast, in case S2 (36-year-old female) who failed to reduce HBsAg titer in the course of a sequential combination therapy, such dynamic changes in CXCL13 and IL-21 during PEG-IFN- $\alpha$ administration were not observed, although a similar increase in CXCL10 and CXCL11 at the beginning of PEG-IFN- $\alpha$ was observed (Figure 4). After the cessation of PEG-IFN- $\alpha$, case S2 developed hepatic flare accompanied with the elevation of CXCL9, CXCL10, and CXCL11. Even at flare in this case, CXCL13 and IL-21 levels were not increased, supporting the notion that CXCL13 and IL-21 elevations are not only caused by inflammatory response. These cases suggest that the activation of the CXCL13 and IL-21 axis, as reflected by the increase in their serum levels, is associated with attaining functional cure in patients with $\mathrm{CH}$.

\section{Discussion}

Functional cure is a desirable clinical target in patients with $\mathrm{CH}$, the probability of which is approximately $9 \%$ in 5 years of a combination therapy with PEG-IFN- $\alpha$ and tenofovir disoproxil fumarate (TDF) (4). In this study, we investigated serum chemokines and cytokines and their potential role in functional cure of HBV. We interrogated cohorts of patients with $\mathrm{AH}$ infection that clear infection or develop persistence, $\mathrm{CH}$ infection, and $\mathrm{CH}$ infection with acute flare as well as HBV-inoculated chimpanzees using a chemokine panel and IL-21 ELISA. We also analyzed 2 patients treated with NAs followed by PEG-IFN- $\alpha$, one resulting in functional cure. We found that CXCL9, CXCL10, CXCL11, CXCL13, and IL-21 were increased at the peak of ALT elevation and decreased together with HBV DNA reduction during acute infection. Secretion of CXCL9, CXCL10, CXCL11, CXCL13, and IL-21 was impaired in $\mathrm{CH}$ patients with persistent infection. Only IL-21 was specifically elevated in patients that continued to resolve acute infection, compared with $\mathrm{AH}$ patients resulting in persistent infection. Induction of CXCL13 and IL-21 was also temporally linked with clearance in the one CH patient treated successfully with antiviral therapy, implying that CXCL13 and IL-21 induction might have some roles in HBsAg loss. A positive correlation of IL-21 with HBeAg or HBsAg seroconversion was reported in

Table 2. Clinical background of the patients in the longitudinal analysis

\begin{tabular}{|c|c|c|c|c|}
\hline Case & Sex & Age (yr) & HBV Genotype & HBeAg/Ab \\
\hline $\mathrm{AH} 1$ & female & 35 & A & $+/-$ \\
\hline $\mathrm{AH} 2$ & male & 47 & $C$ & $+/-$ \\
\hline $\mathrm{AH} 3$ & male & 39 & A & $+1-$ \\
\hline $\mathrm{AH} 4$ & male & 35 & $A$ & $+/-$ \\
\hline
\end{tabular}




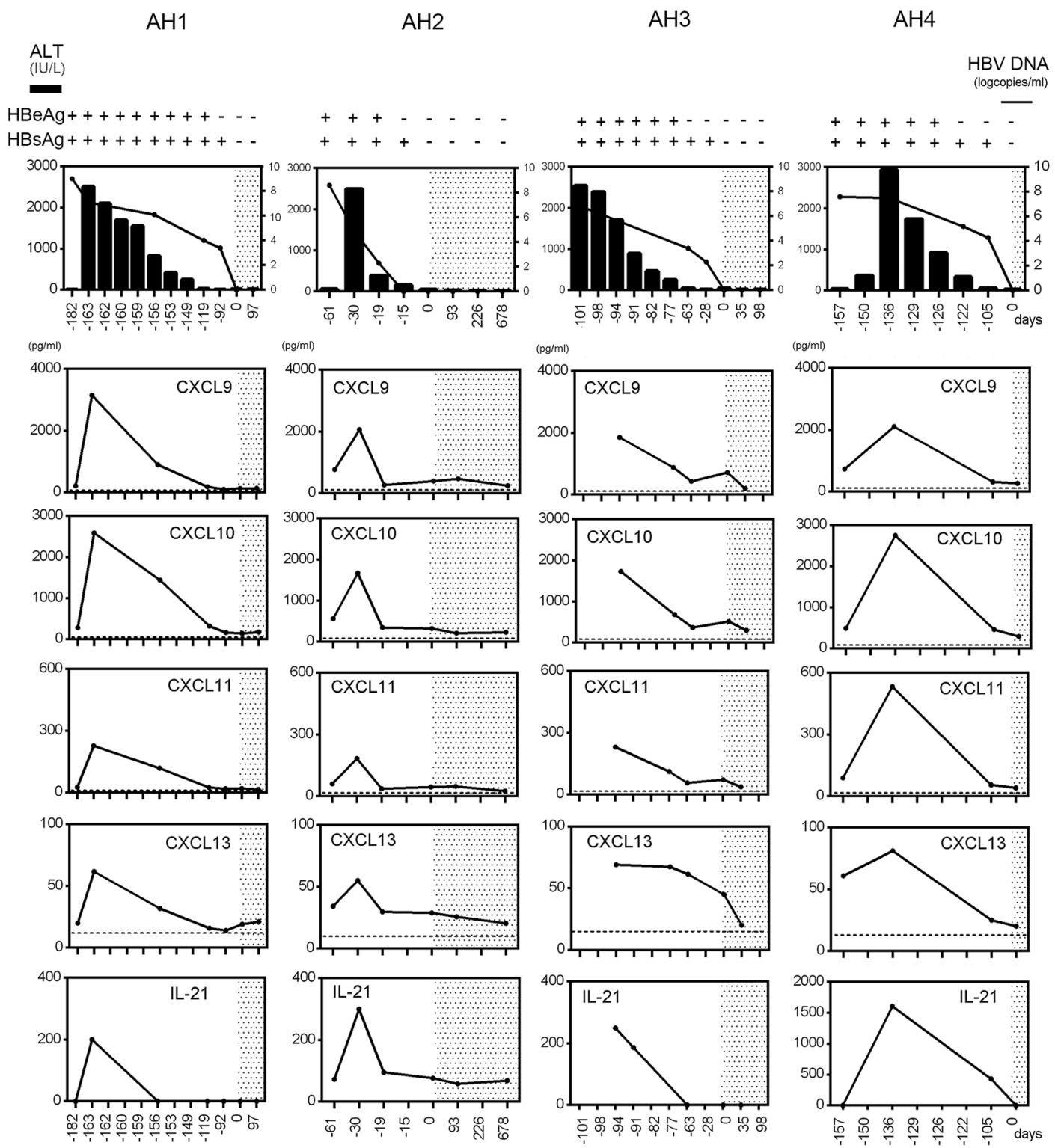

Figure 2. Longitudinal and comparative analysis of CX3CL1, CXCL9, CXCL10, CXCL11, CXCL13, and IL-21 in patients with self-limited HBV infection. Four representative cases are shown (AH1-AH4). Dotted lines in the panels indicate the average chemokine concentration in healthy volunteers. The shaded area depicts the time period of HBsAg negativity. In top panels, the left vertical axes are for alanine aminotransferase (ALT, IU/I), and the right vertical axes are for HBV DNA (log copies/ml).

$\mathrm{CH}$ patients who underwent NA therapy $(15,16)$. In support of these reports, we showed here a case of $\mathrm{CH}$ who attained HBsAg seroconversion by a sequential therapy (Figure 4). Serum levels of CXCL13 and IL-21 were significantly increased after the beginning of PEG-IFN- $\alpha$ in parallel with HBsAg loss, suggesting some responsiveness of IFN- $\alpha$ in the production of CXCL13 or subsequent IL-21.

IL-21 is expressed mainly on activated human $\mathrm{CD}^{+} \mathrm{T}$ cells, such as $\mathrm{T}$ follicular helper (Tfh) cells (17) and Th17 cells (18). IL-21 regulates differentiation, proliferation, and activation of T cells, B cells, or NK cells (19). Publicover et al. first discovered the role for IL-21 in HBV clearance using a mouse model, and confirmed relevance to human disease by showing that patients who clear HBV have higher IL-21 expression in peripheral blood mononuclear cells compared with $\mathrm{CH}$ patients and uninfected controls (20). They also showed that IL-21 production by Tfh cells facilitates IgG class switching in B cells and diversification of HBV-specific T cell response including increased IFN- $\gamma$ production (20). In a mouse model of in vivo $\mathrm{HBV}$ transduction, it is also reported that activation of intrahepatic $\mathrm{CD} 4^{+}(\mathrm{Tfh})$ cells and their relevant signaling molecules, including IL-21, was associated with HBV clearance (21). 
Table 3. Clinical background of the chimpanzees in the longitudinal analysis

\begin{tabular}{ccccccccc}
\hline Chimp & Age (years) & Sex & Genotype & $\begin{array}{c}\text { Virus Inoculum } \\
\text { (copies) }\end{array}$ & $\begin{array}{c}\text { ALT levels at peakHBV DNA negative } \\
\text { (IU/ml) }\end{array}$ & $\begin{array}{c}\text { HBsAg loss } \\
\text { (weeks) }\end{array}$ & $\begin{array}{c}\text { HBsAb positive } \\
\text { (weeks) }\end{array}$ \\
(weeks)
\end{tabular}

The forced expression of IL-21 enhanced clearance of preestablished HBV (22). CD4 $4^{+} \mathrm{T}$ cell-derived IL-21 plays an important role in sustaining effector function of $\mathrm{CD}^{+} \mathrm{T}$ cells during chronic viral infection $(23,24)$. Further investigation of the mechanisms underlying the induction of IL-21 and how IL-21 achieves functional cure might lead to developing new anti-HBV drugs.

CXCL9, CXCL10, and CXCL11 are selective ligands for CXCR3. The ligands are usually expressed at low levels in homeostatic conditions, but upregulated by IFN- $\gamma$, IFN- $\alpha / \beta$, or TNF- $\alpha$. CXCR3 is highly expressed on activated T cells, memory T cells, and NK cells. CXCL9 is induced by IFN- $\gamma$ but not by IFN- $\alpha / \beta$ (25). CXCL10 is strongly induced by IFN- $\gamma$ as well as IFN- $\alpha / \beta$ (26). CXCL11 is induced by IFN- $\gamma$ and IFN- $\beta$, and weakly by IFN- $\alpha$ (27). As for the mechanism of CXCL9, CXCL10, and CXCL11 induction, we previously reported that CXCL9, CXCL10, and CXCL11 are induced by HBV-positive hepatocytes with coexisting NK cells and pDCs, which synergistically produce IFN- $\alpha$ and IFN- $\gamma$ in response to HBV (13). A positive correlation among CXCL9, CXCL10, and CXCL11 levels observed in patients with $\mathrm{AH}$ suggests a causal link to immune cells responding to these chemokines.

An increase in CXCL13 at hepatitis was seen in AH patients and HBV GT-C-infected chimpanzees (Ch1, Ch2). Several reports published recently showed that CXCL13 and macrophages are involved in the prevention of HBV transmission and its persistence. Publicover et al. reported that agedependent HBV clearance in a mouse model of hepatitis B is mainly attributable to CXCL13 expression by hepatic macrophages (14). The critical role of macrophages in the fetal liver in the prevention of mother-to-child transmission of HBV has been reported in a mouse model (28). Moreover, a genetic variant of the CXCL13 gene is associated with susceptibility to intrauterine infection by HBV (27-29). In response to CXCL13, mature B cells and Tfh cells $(30,31)$, which express the receptor CXCR5, could be subsequently attracted to the liver and produce HBV-specific antibodies. A positive correlation of HBV DNA titer with CXCL13 was observed in patients with resolving AH (Figure 1C), while CXCL13 was not elevated in $\mathrm{CH}$ patients who failed to attain HBsAg loss, even at flare (Figure 1A). These data might suggest the important role of Tfh cells in HBsAg loss.

In summary, we showed here the dynamics of serum cytokines and chemokines in resolving patients with $\mathrm{AH}$ or in a $\mathrm{CH}$ patient who gained HBsAg loss after treatment. Activation of CXCL9, CXCL10, CXCL11, CXCL13, and IL-21 response might be a signature pattern in attaining functional HBV cure.

\section{Methods}

\section{Study design and sample collection}

Patients. Adult $\mathrm{AH}$ and $\mathrm{CH}$ patients who were followed in Kohnodai Hospital and Osaka National Hospital were enrolled in this study. For cross-sectional analysis, $41 \mathrm{sAH}$ patients who achieved HBsAg loss with antiHBs antibody within 6 months of diagnosis, $8 \mathrm{AH}$ patients who failed to achieve HBsAg loss (pAH), and $8 \mathrm{CH}$ patients with the absence of HBeAg who experienced hepatic flare were studied (Table 1). Fourteen healthy uninfected people were analyzed as controls. The pAH group was defined as patients who were $\mathrm{HBsAg}$ positive more than 1 year after they were diagnosed as $\mathrm{AH}$. They were all anti-HBc IgM greater than $10 \mathrm{~S} / \mathrm{CO}$ (sample/cut-off). Not less than $1 \mathrm{~S} / \mathrm{CO}$ is positive for anti-HBc IgM. Hepatic flare was defined as elevation of ALT of more than 5 times the upper limit of the normal range (32). The levels of ALT, HBV DNA, chemokines, and cytokines in $\mathrm{CH}$ patients were compared between those at stable conditions and those at hepatic flare. For longitudinal analysis, we enrolled 4 patients with $\mathrm{AH}$ who accomplished $\mathrm{HBsAg}$ loss with anti-HBs antibody (Figure 2 and Table 2) and 2 patients who had been treated with a sequential 
ALT

IU/L

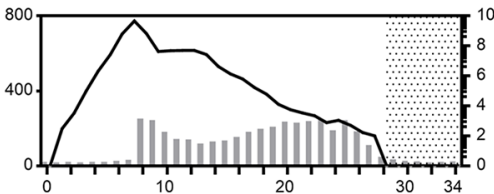

$\mathrm{U} / \mathrm{mL}$
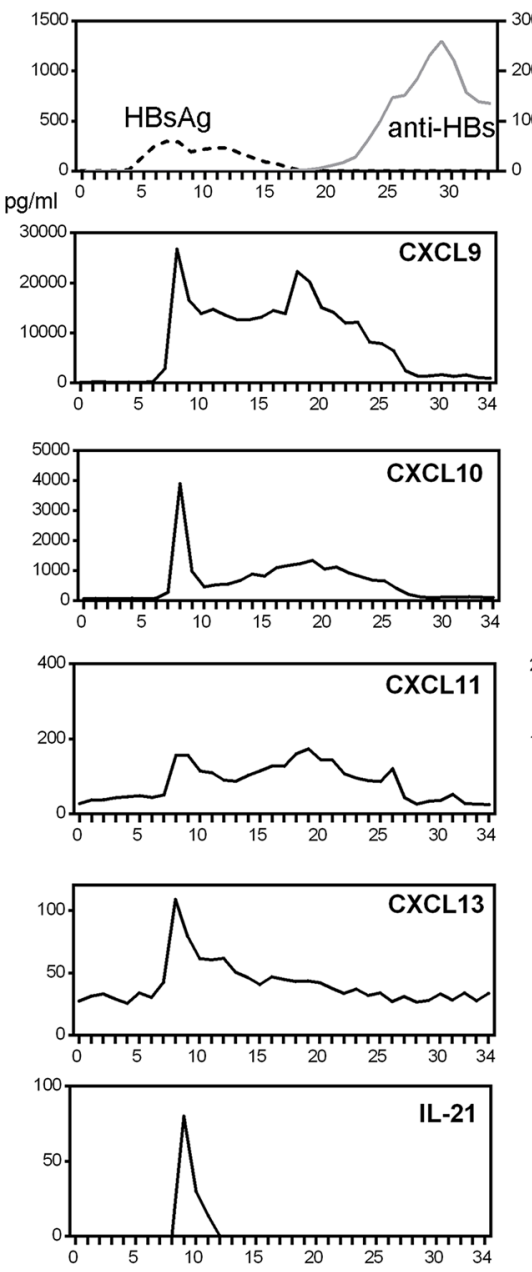

Ch2
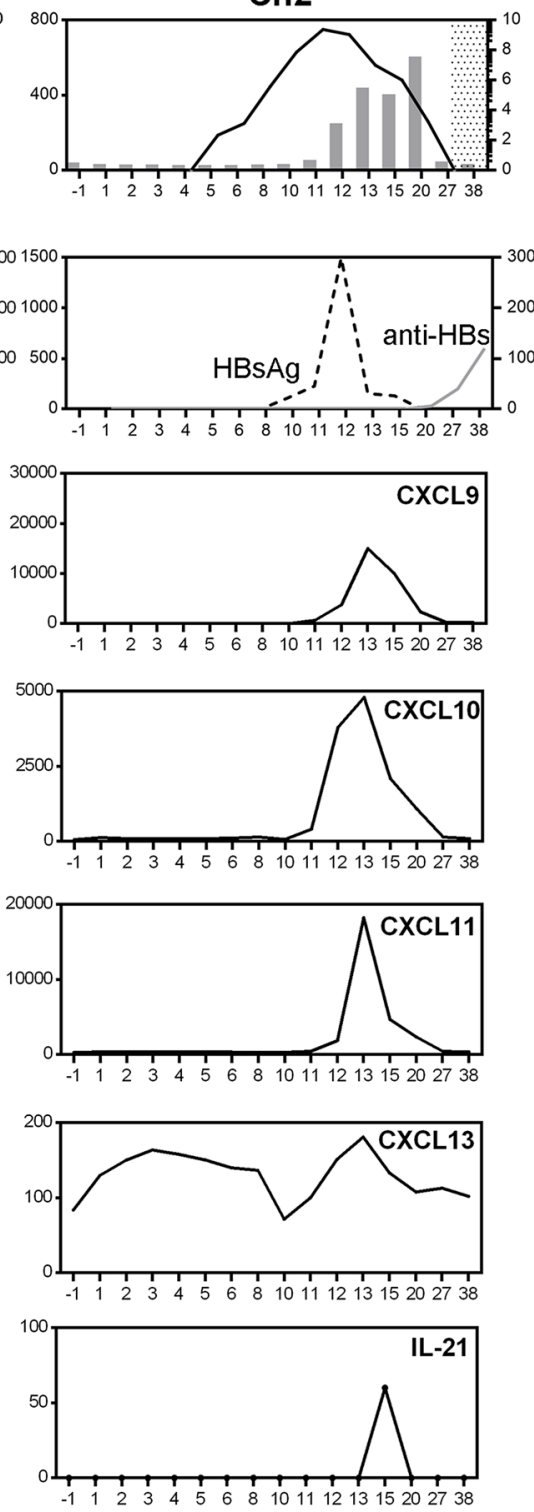

HBVDNA
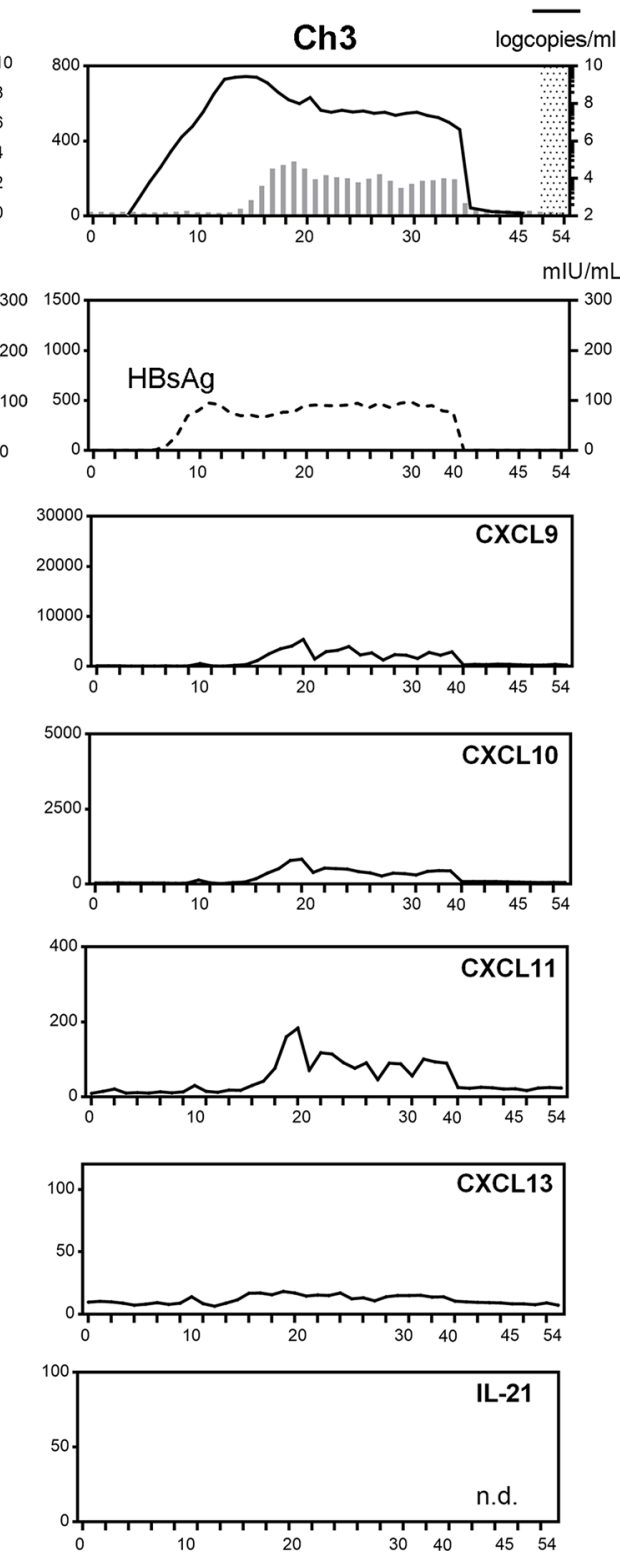

Figure 3. Sequential analyses of serum chemokines in HBV-inoculated chimpanzees. The left vertical axes are for ALT (bar charts), HBsAg (dotted lines) and anti-HBs (solid gray lines), and the right vertical axes are for HBV DNA(solid black lines). In top panels, the shaded area depicts the time period of HBV DNA negativity. n.d., not detected.

combination of NAs followed by 48 weeks of PEG-IFN- $\alpha$ (Figure 4). All the enrolled patients were negative for hepatitis $\mathrm{C}$ virus (HCV), hepatitis delta virus (HDV), and human immunodeficiency virus (HIV). $\mathrm{HBsAg}$ was quantified using Architect HBsAg QT (Abbott). HBV DNA was quantified by quantitative real-time PCR using the COBAS TaqMan HBV test (Roche Diagnostics). Anti-HDV IgM was tested using AccuDiag HDV IgM ELISA (Diagnostic Automation).

\section{Chimpanzees (Pan troglodytes)}

Three healthy adult chimpanzees, Ch1 (high-titer genotype C [GT-C]), Ch2 (low-titer GT-C), and Ch3 (high-titer GT-A) were studied for the analysis of the minimum infectious dose and early dynamics of HBV at Sanwa Kagaku Kenkyusho Co., Ltd, conducted by Hiroshima University in 2003 (33) (Table 3). None of the 3 chimpanzees had serologic or molecular biologic evidence of past or present HBV infection prior to the inoculation. Blood samples were drawn from them after the inoculation while they were under anesthesia 

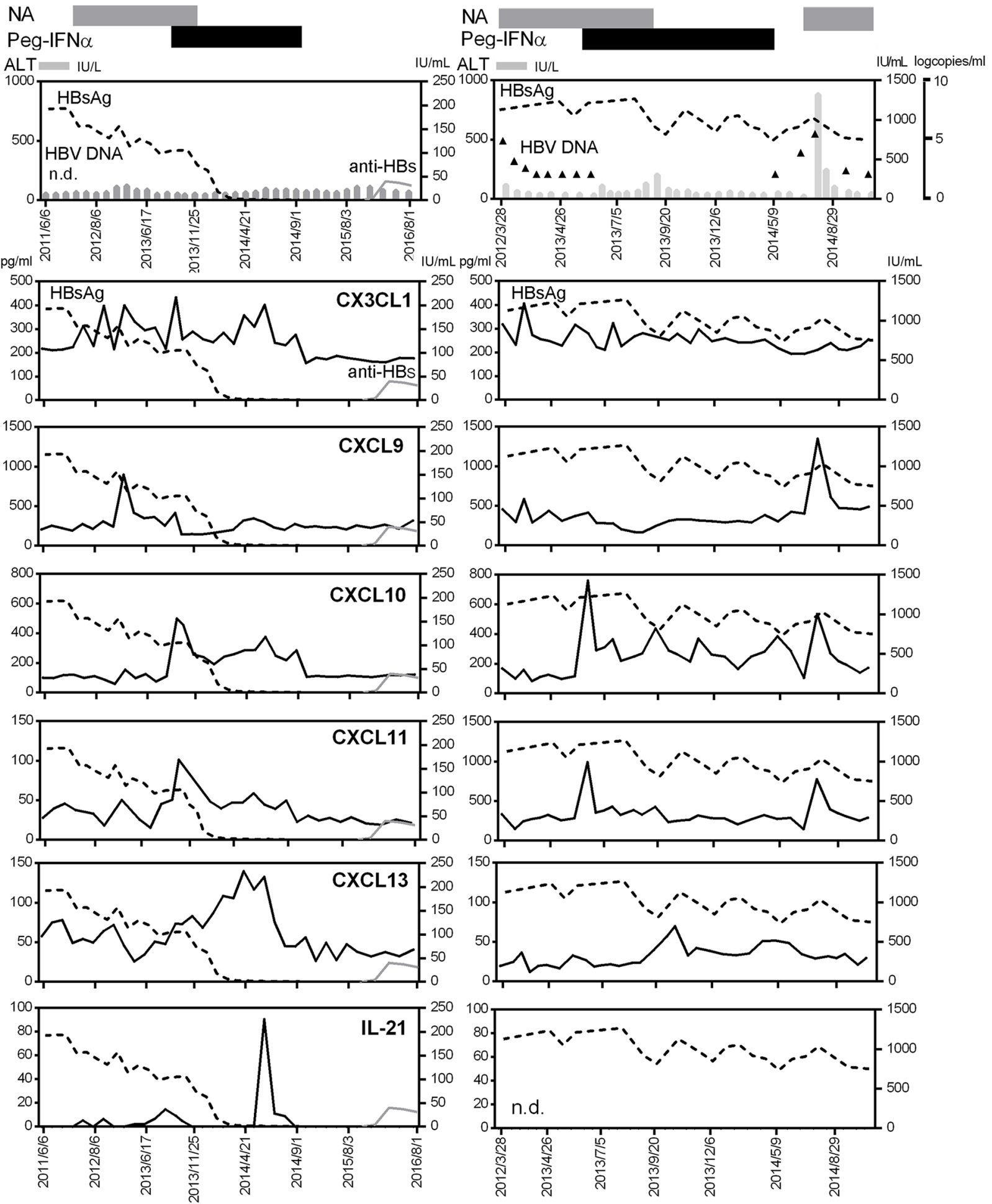

Figure 4. Sequential analyses of serum chemokines in chronic hepatitis patients with a sequential therapy of nucleoside analogues and PEG-IFN- $\alpha$. Case S1 (50 years old, male) was HBsAg positive (HBsAg titer $156 \mathrm{lU} / \mathrm{ml}$, genotype B), HBeAg negative, and HBeAb positive at the start of a sequential therapy consisting of a combination of nucleoside analogs (NAs) and subsequent PEG-IFN- $\alpha$ ( 48 weeks). Case S2 (36 years old, female) was HBsAg positive (HBsAg titer 1,130 IU/ml, genotype C), $\mathrm{HBeAg}$ negative, and $\mathrm{HBeAb}$ positive at the start of the sequential therapy. At the end of treatment, HBV DNA was negative but HBsAg was $876 \mathrm{IU} / \mathrm{ml}$. Two months after the end of treatment, case $\mathrm{S} 2$ had hepatic flare (HBV DNA 5.5 log copies/ml, ALT 585 IU/I). The durations of NA and PEG-IFN- $\alpha$ treatments in cases S1 and S2 are shown as gray and black bars at the top of the panels. The left vertical axes are for ALT (bar chart), CX3CL1, CXCL9, CXCL10, CXCL11, CXCL13, and IL-21 (solid black line). The right vertical axes are for HBsAg titers (dotted line) and anti-HBs (solid gray line). The additional right vertical axis is for HBV DNA titers (triangles) (case S2). n.d., not detected. 
with ketamine hydrochloride. The chimpanzees were studied under an animal study protocol. All were kept in individual cages and received humane care in accordance with all relevant requirements for the use of primates in the facility approved by the Ethics Committee.

\section{Chemokine secretion assays}

Serum samples were prepared by centrifugation at $300 \mathrm{~g}$ for 15 minutes and stored at $-80^{\circ} \mathrm{C}$. A Bio-Plex Pro Human Chemokine Panel (40 plex) (Bio-Rad) was used per the manufacturer's instructions. The complete panel was screened for the expression of the following: CXCL9 (MIG), CXCL10 (IP-10), CXCL11 (I-TAC), CXCL13 (BCA1), CCL1 (I-309), CCL2 (MCP-1), CCL3 (MIP-1 $\alpha$ ), CCL7 (MCP-3), CCL8 (MCP-2), CCL11

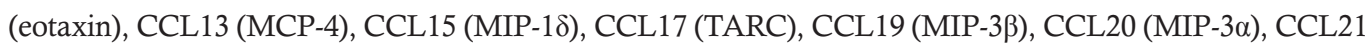
(6Ckine), CCL22 (MDC), CCL23 (MPIF1), CCL24 (eotaxin-2), CCL25 (TECK), CCL26 (eotaxin-3), CCL27 (CTACK), CXCL1 (Gro- $\alpha$ ), CXCL2 (Gro- $\beta$ ), CXCL5 (ENA-78), CXCL6 (GCP-2), CXCL12 (SDF$1 \alpha+\beta)$, CXCL16 (SCYB16), CX3CL1 (Fractalkine), GM-CSF, MIF, TNF- $\alpha$, IFN- $\gamma$, IL-1b, IL-2, IL-4, IL-6, IL-8, IL-10, and IL-16. IL-21 was measured by ELISA (eBioscience). The sera from chimpanzees were examined using human assay systems. Given the extremely close evolutionary relationship, chimpanzee proteins are, on average, more than $99 \%$ identical to human proteins (34). Values of more than 2 times above the upper limit of the normal range or the baseline of each individual were defined as being elevated.

\section{Statistics}

Mann-Whitney nonparametric $U$ test and box-and-whisker plots were used for comparisons among HV, $\mathrm{sAH}, \mathrm{pAH}, \mathrm{CH}$, and $\mathrm{CH}$-at-flare groups. Spearman's rank correlation coefficients were used for analyzing correlations among the levels of chemokines, cytokines, HBsAg, HBV DNA, and ALT. GraphPad Prism software was used, and a $P$ value less than 0.05 was considered significant.

\section{Study approval}

The study was approved by the Ethics Committees of the National Center for Global Health and Medicine (Tokyo, Japan) (NCGM-G-001839-00) and the National Hospital Organization National Osaka Hospital (0956) (Osaka, Japan). Written informed consent was received from the participants prior to their inclusion in the study.

\section{Author contributions}

SY and TK designed the research studies. SY conducted experiments and acquired data. SY and TK wrote the manuscript. YM, HD TS, HS, MM, TM, HK, YS, YO, MS, MM, MK, EM, KK, and JT collected samples and provided critical reviews of the manuscripts.

\section{Acknowledgments}

This study was supported by grants from the National Center for Global Health and Medicine (26A201, 29-shi-1011) and grants from the Program for Basic and Clinical Research on Hepatitis (JP17fk0310119h, JP17fk0310105h, and JP17fk0310106h). We are grateful to Chizu Kanokoda for technical assistance.

Address correspondence to: Tatsuya Kanto, The Research Center for Hepatitis and Immunology, National Center for Global Health and Medicine, 1-7-1, Kohnodai, Ichikawa 272-8516, Japan. Phone: 81.47.372.3501; Email: kantot@hospk.ncgm.go.jp.

1. [No authors listed]. Global Hepatitis Report, 2017. World Health Organization. http://www.who.int/hepatitis/publications/ global-hepatitis-report2017/en/. Accessed September 17, 2018.

2. Trépo C, Chan HL, Lok A. Hepatitis B virus infection. Lancet. 2014;384(9959):2053-2063.

3. Zoulim F, Durantel D. Antiviral therapies and prospects for a cure of chronic hepatitis B. Cold Spring Harb Perspect Med. 2015;5(4):a021501.

4. Marcellin P, et al. Regression of cirrhosis during treatment with tenofovir disoproxil fumarate for chronic hepatitis B: a 5-year open-label follow-up study. Lancet. 2013;381(9865):468-475.

5. Zeisel MB, et al. Towards an HBV cure: state-of-the-art and unresolved questions--report of the ANRS workshop on HBV cure. Gut. 2015;64(8):1314-1326.

6. Durantel D, Zoulim F. New antiviral targets for innovative treatment concepts for hepatitis B virus and hepatitis delta virus. $J$ Hepatol. 2016;64(1 Suppl):S117-S131. 
7. Lucifora J, et al. Specific and nonhepatotoxic degradation of nuclear hepatitis B virus cccDNA. Science. 2014;343(6176):1221-1228.

8. Guidotti LG, Rochford R, Chung J, Shapiro M, Purcell R, Chisari FV. Viral clearance without destruction of infected cells during acute HBV infection. Science. 1999;284(5415):825-829.

9. Asabe S, et al. The size of the viral inoculum contributes to the outcome of hepatitis B virus infection. J Virol. 2009;83(19):9652-9662.

10. Thimme R, et al. CD8(+) T cells mediate viral clearance and disease pathogenesis during acute hepatitis B virus infection. $J$ Virol. 2003;77(1):68-76.

11. Webster GJ, et al. Incubation phase of acute hepatitis B in man: dynamic of cellular immune mechanisms. Hepatology. 2000;32(5):1117-1124.

12. Fisicaro P, et al. Early kinetics of innate and adaptive immune responses during hepatitis B virus infection. Gut. 2009;58(7):974-982

13. Yoshio S, et al. Indoleamine-2,3-dioxygenase as an effector and an indicator of protective immune responses in patients with acute hepatitis B. Hepatology. 2016;63(1):83-94.

14. Publicover J, et al. Age-dependent hepatic lymphoid organization directs successful immunity to hepatitis B. J Clin Invest. 2013;123(9):3728-3739.

15. Li Y, et al. Circulating chemokine (C-X-C Motif) receptor 5(+) CD4(+) T cells benefit hepatitis B e antigen seroconversion through IL-21 in patients with chronic hepatitis B virus infection. Hepatology. 2013;58(4):1277-1286.

16. Vyas AK, Sharma BC, Sarin SK, Trehanpati N. Immune correlates of hepatitis B surface antigen spontaneous seroconversion in hepatitis B e antigen negative chronic hepatitis B patients. Liver Int. 2018;38(1):38-49.

17. Chtanova T, et al. T follicular helper cells express a distinctive transcriptional profile, reflecting their role as non-Th1/Th2 effector cells that provide help for B cells. J Immunol. 2004;173(1):68-78.

18. Wei L, Laurence A, Elias KM, O'Shea JJ. IL-21 is produced by Th17 cells and drives IL-17 production in a STAT3-dependent manner. J Biol Chem. 2007;282(48):34605-34610.

19. Ettinger R, et al. IL-21 induces differentiation of human naive and memory B cells into antibody-secreting plasma cells. $J$ Immunol. 2005;175(12):7867-7879.

20. Publicover J, et al. IL-21 is pivotal in determining age-dependent effectiveness of immune responses in a mouse model of human hepatitis B. J Clin Invest. 2011;121(3):1154-1162.

21. Song XF, et al. Activation of intrahepatic $\mathrm{CD} 4^{+} \mathrm{CXCR} 5^{+} \mathrm{T}$ and $\mathrm{CD} 19^{+} \mathrm{B}$ cells is associated with viral clearance in a mouse model of acute hepatitis B virus infection. Oncotarget. 2016;7(32):50952-50962.

22. Shen Z, et al. Hepatitis B virus persistence in mice reveals IL-21 and IL-33 as regulators of viral clearance. Nat Commun. 2017;8(1):2119.

23. Xin G, et al. A critical role of IL-21-induced BATF in sustaining CD8-T-cell-mediated chronic viral control. Cell Rep. 2015;13(6):1118-1124

24. Salimzadeh L, et al. PD-1 blockade partially recovers dysfunctional virus-specific B cells in chronic hepatitis B infection. J Clin Invest. 2018;128(8):4573-4587.

25. Farber JM. Mig and IP-10: CXC chemokines that target lymphocytes. J Leukoc Biol. 1997;61(3):246-257.

26. Qian C, An H, Yu Y, Liu S, Cao X. TLR agonists induce regulatory dendritic cells to recruit Th1 cells via preferential IP-10 secretion and inhibit Th1 proliferation. Blood. 2007;109(8):3308-3315.

27. Rani MR, Foster GR, Leung S, Leaman D, Stark GR, Ransohoff RM. Characterization of beta-R1, a gene that is selectively induced by interferon beta (IFN-beta) compared with IFN-alpha. J Biol Chem. 1996;271(37):22878-22884.

28. Tian Y, Kuo CF, Akbari O, Ou JH. Maternal-derived hepatitis B virus e antigen alters macrophage function in offspring to drive viral persistence after vertical transmission. Immunity. 2016;44(5):1204-1214.

29. Wan Z, et al. Genetic variant in CXCL13 gene is associated with susceptibility to intrauterine infection of hepatitis B virus. $S c i$ Rep. 2016;6:26465.

30. Legler DF, Loetscher M, Roos RS, Clark-Lewis I, Baggiolini M, Moser B. B cell-attracting chemokine 1, a human CXC chemokine expressed in lymphoid tissues, selectively attracts B lymphocytes via BLR1/CXCR5. J Exp Med. 1998;187(4):655-660.

31. Förster R, Emrich T, Kremmer E, Lipp M. Expression of the G-protein--coupled receptor BLR1 defines mature, recirculating B cells and a subset of T-helper memory cells. Blood. 1994;84(3):830-840.

32. Chang ML, Liaw YF. Hepatitis B flares in chronic hepatitis B: pathogenesis, natural course, and management. $J$ Hepatol. 2014;61(6):1407-1417.

33. Matsubara N, et al. A novel hepatitis B virus surface antigen immunoassay as sensitive as hepatitis B virus nucleic acid testing in detecting early infection. Transfusion. 2009;49(3):585-595.

34. Chimpanzee Sequencing Analysis Consortium. Initial sequence of the chimpanzee genome and comparison with the human genome. Nature. 2005;437(7055):69-87. 